\title{
Design and optimization of full decoupled micro/nano-positioning stage based on mathematical calculation
}

\author{
Zhigang $\mathrm{Wu}^{1}$, Yangmin $\mathrm{Li}^{2}$, and $\mathrm{Min} \mathrm{Hu}^{1}$ \\ ${ }^{1}$ The School of Energy and Machinery Engineering, \\ Jiangxi University of Science and Technology, Nanchang 330013, China \\ ${ }^{2}$ Department of Industrial and Systems Engineering, \\ The Hong Kong Polytechnic University, Hong Kong, Hong Kong
}

Correspondence: Zhigang Wu (wzgang2017@ 163.com)

Received: 11 July 2018 - Revised: 4 November 2018 - Accepted: 12 November 2018 - Published: 21 November 2018

\begin{abstract}
Nano-positioning is widely used in Micro-electromechanical Systems (MEMS), micromanipulator and biomedicine, coupling errors and tiny output displacements are the main disadvantages of the one. A totally uncoupled micro/nano-positioning stage with lever amplifiers is designed and tested in this paper. It is fully symmetrical along with the $x$ - and $y$-directions. For obtaining large output displacements, two fully symmetric two-stage lever displacement amplifiers are utilized to amplify output displacements of piezoelectric actuators (PZTs). The established models for performances evaluation of the stage, in terms of kinetostatics, amplification ratio, reachable workspace, the input and output stiffness, are verified by finite element analysis (FEA). After that, the dimensional optimization is also carried out through the genetic optimization algorithm. The prototype of the mechanism is fabricated by using Wire-Electrical-Discharge-Machining (WEDM) process. Testing results indicate that the proposed micromanipulator demonstrates good performance.
\end{abstract}

\section{Introduction}

Flexible hinge, which possesses these advantages of no backlash, no friction, simple structure, and easy manufacture, is widely applied in the micromanipulation system including micro/nano-positioning stages, micromanipulators, and high-accuracy alignment instruments (Tian et al., 2009). Micromanipulation system has been paid more and more attention in recent years, especially the parallel compliant mechanisms own some inherent advantages as big load capacity, high velocity, and high precision compared with serial ones (Dong et al., 2016). Piezoelectric actuators (PZTs) are usually selected as the actuators of the compliant mechanisms due to these advantages of fast response and high precision (Yong et al., 2009). Unfortunately, small output displacements of the PZTs and cross coupling motions of the endeffector have badly restricted the further application (Yu et al., 2011) and development of the compliant mechanisms (Yu et al., 2015).
At present, some amplifiers between the actuator and motion stage have also been proposed to overcome the disadvantage of small output displacement. Such as the lever displacement amplifiers (Tang and $\mathrm{Li}, 2015$ ) and bridge-type amplifiers (Wu and $\mathrm{Li}, 2014)$ are commonly used as the bridge for amplifying the output displacements.

Cross coupling of compliant mechanisms at $x$ - and $y$ directions is an inevitable property in parallel mechanism (Dong et al., 2007). It can seriously influence the precision of motion (Hao et al., 2016). The 2-degree-of-freedom (2DOF) compliant stages include two types of structure: One is in series and another is in parallel (Fan et al., 2018). For example, Lin and Lin (2012) have designed a series of $x y$ compliant mechanisms with the maximal cross coupling error of $0.12 \mu \mathrm{m}$ and the frequency of $50 \mathrm{~Hz}$. Additionally, Polit and Dong (2011) have also proposed a high-bandwidth $x y$ positioning stage with the cross coupling motion of $0.2 \%$ at the total workspace of $15 \mu \mathrm{m} \times 15 \mu \mathrm{m}$ in two directions. A kind of novel $x y$ micromanipulation stage for micro/nano 
positioning and manipulation is also developed by Qin et al. (2013), which has the workspace of $8 \mu \mathrm{m} \times 8 \mu \mathrm{m}$ with the first natural frequency of $665.4 \mathrm{~Hz}$, while the cross-axis coupling error is $2 \%$ more than previous results. In addition, Liu et al. (2015) have also designed a novel $x y$ parallel compliant mechanism with the first natural frequency of $763.23 \mathrm{~Hz}$ more than other papers.

Based on the aforementioned analyses, the micromanipulation stage is proposed in this paper with low cross coupling, large reachable workspace, high stiffness and high bandwidth. It aims to improve the positioning precision of micromanipulator. The theoretical calculation, simulation analysis, and prototype tests demonstrate that the presented 2DOF micromanipulator with mechanical amplifiers owns the performance for cross coupling error of under $0.1 \%$ at the full range of $169.6 \mu \mathrm{m} \times 165.3 \mu \mathrm{m}$, with the frequency of $348.31 \mathrm{~Hz}$, which can be used in high frequency positioning micromanipulation system. Additionally, the positioning system will be controlled easily by reducing the coupling errors, which have the important applying value in the field of micro-nano manufacturing platform or micro 3-D printing system combining with the micro gripper. The main contribution of this paper is described as follows: (a) the structural design, kinematics and statics modeling analysis, and prototype test of this novel mechanism; (b) the lower cross coupling errors with larger workspace compared with other papers.

The remainder of this paper is organized as follows. The concept of mechanical amplifier and the process of mechanism design is described in Sect. 2. Then in Sect. 3, the kinematics and dynamics analyses of the mechanism with amplification ratio, stiffness, reachable workspace and natural frequency are presented in details. Besides, the performance evaluation and model verification implemented by the finite element analysis (FEA) are conducted in Sect. 4. Afterwards, structure optimization is carried out through genetic optimization algorithm in Sect. 5. Furthermore, prototype fabrication and performance tests are presented in Sect. 6. Finally, the conclusions and future work are presented in Sect. 7.

\section{Structural design}

\subsection{The concept design of lever amplifier}

Lever amplifier based on flexure hinges possesses the advantages of high amplification ratio and simple structure (Choi et al., 2007). A two-stage amplifier is shown in Fig. 1.

According to the mechanical principle, supposing that an outside force $F_{\text {in }}$ is applied at free-end, the corresponding deformation displacement is $\delta$. Therefore, output displacements $d_{\text {out }}$ and $\delta_{\text {out }}$ are obtained. Considering Fig. 1, it can be observed that the two-stage amplifier is composed of two one-stage amplifiers. Assuming that the input displacement of two-stage magnifier is also $\delta$, the total theoretical magnification ratio can be written by

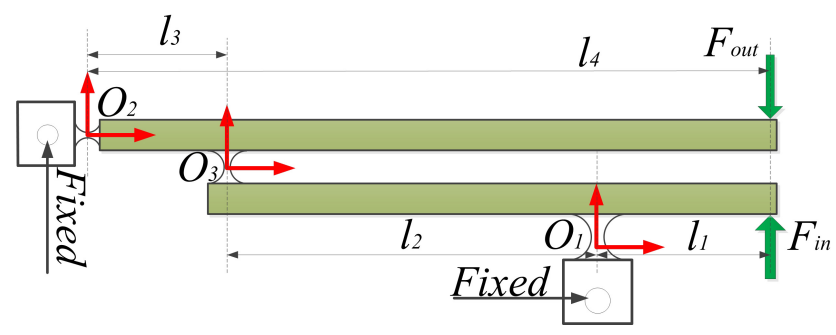

Figure 1. The two-stage amplifier.

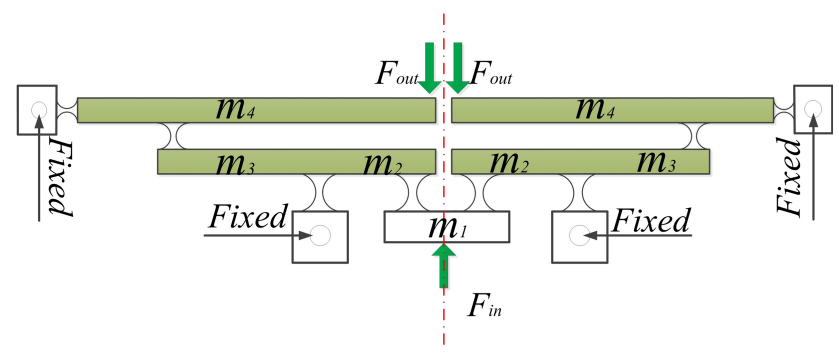

Figure 2. Symmetrical structure with two 2-stage amplifiers.

$\mathrm{AR}=\mathrm{AR}_{1} \times \mathrm{AR}_{2}=\frac{l_{2}}{l_{1}} \times \frac{l_{4}}{l_{3}}$

For enhancing the input stiffness, the parallel structure is used as an amplification mechanism for amplifying the input displacement as shown in Fig. 2. The mechanism is fully symmetric along with the central line and is connected by two amplifiers in parallel. The symmetrical structure of input-end of the mechanism can protect the PZT from damage.

\subsection{2-DOF positioning stage}

To eliminate cross coupling of the end-effector, symmetrical 1-DOF structure is shown in Fig. 3, where the double fourbar parallelogram mechanism is designed to connect the motion stage. The $4 \mathrm{P}$-joint is composed of the four prismatic joints.

Simplified principle diagram of the 2-DOF positioning stage is presented as shown in Fig. 4, where the four mechanical displacement amplifiers and two double four-bar parallelogram mechanisms are utilized to design a fully symmetrical mechanism along with $x$ - and $y$-directions. Particularly, the vertical distribution structure of two double four-bar mechanisms can effectively decrease cross coupling.

After a series of elaborated designs, three-dimension (3D) model of the mechanism driven by PZT is proposed as shown in Fig. 5, input displacements of the motion platform, which are conducted by the PZTs installed in the $x$ - and $y$ directions, can be magnified by the displacement amplifiers. Due to the symmetry of the structure and the four-bar parallelogram, parasitic motions of the positioning stage can be 


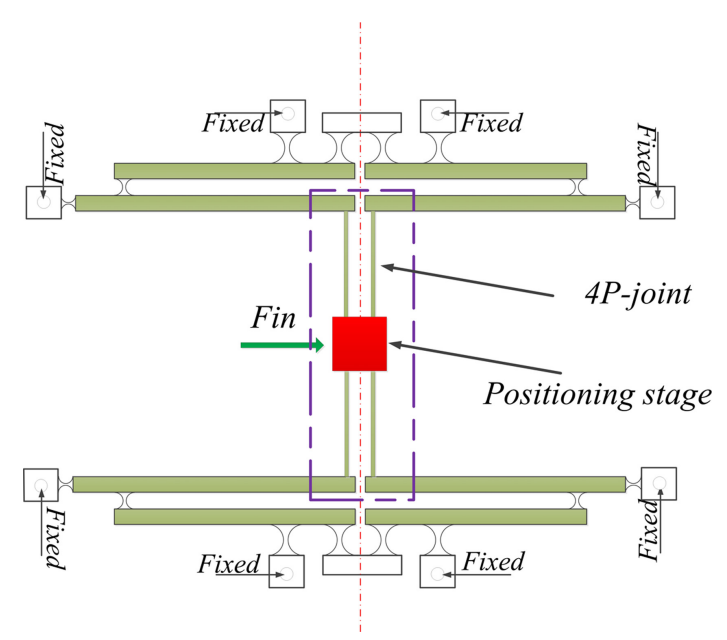

Figure 3. 1-DOF symmetrical mechanism.

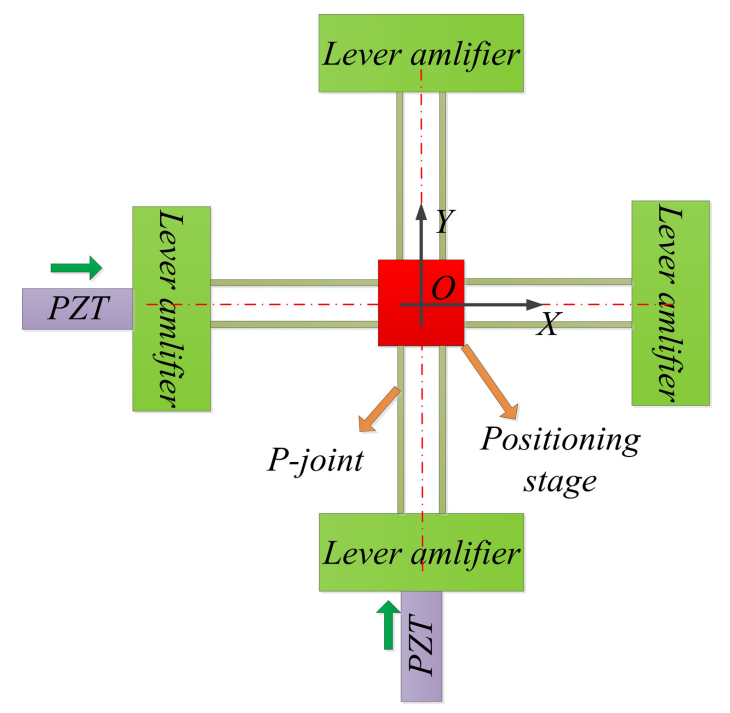

Figure 4. Simplified principle diagram of mechanism.

reduced or even eliminated. Circular hinges are adopted in the mechanical amplifier to avoid the parasitic motions.

\section{Kinetostatics and dynamics modeling analysis}

There are many modeling methods to analyze the kinematic performance of compliant mechanism, such as the numerical model method, the pseudo-rigid-body (PRB) model method, and the compliance matrix model method (Tang and $\mathrm{Li}$, 2013). In this section, kinetostatics and dynamics modeling of the compliant mechanism, including the amplification ratio (AR), input stiffness, reachable workspace and natural frequency, are analyzed by the numerical modeling method and Lagrange's method.

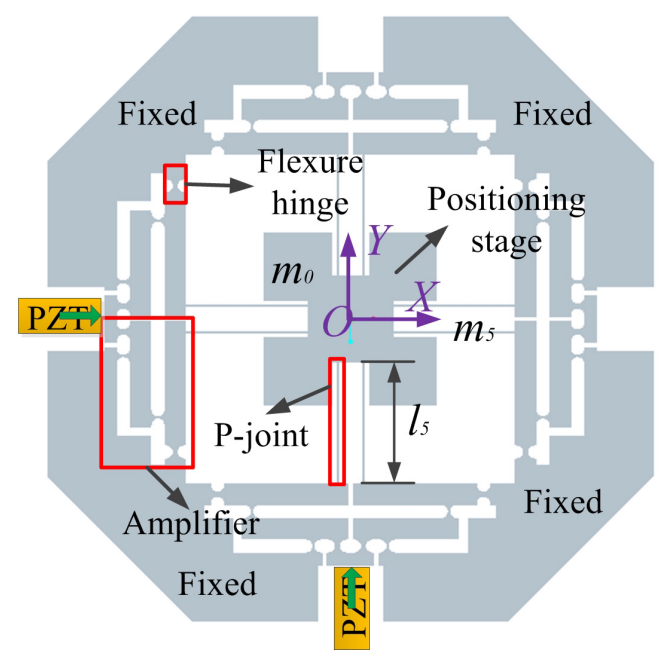

Figure 5. The 3-D diagram of the 2-DOF compliant mechanism.

\subsection{Amplification ratio and input stiffness analysis}

Firstly, the amplification ratio and input stiffness of the mechanism are analyzed. The flexure circular notched hinge can be regarded as the general spring with bending stiffness $\left(k_{\mathrm{b}}\right)$ created by lateral force, torsion stiffness $\left(k_{\mathrm{t}}\right)$ caused by the torque and linear stiffness $\left(k_{1}\right)$ produced by the axial force. Additionally, $k_{\mathrm{t}}^{\prime}$ and $k_{1}^{\prime}$ are the torsion stiffness and linear stiffness of the prismatic beam (Koseki et al., 2002). Therefore, the rotational and linear deformations may simultaneously occur when a force is applied at the free-end of the beam. As shown in Fig. 6, the simplified diagrams of the circular notched hinge and prismatic beam are presented. Additionally, the torque $M_{\mathrm{t}}$ is produced after a small deformation angle $\Delta \theta$ occurs along with the center of the hinge from inside of the flexure hinge. Therefore, stiffness of different directions for flexure hinge can be represented in the following equations:

$$
\begin{cases}k_{\mathrm{t}}=\frac{2 E b t^{2.5}}{9 \pi r^{0.5}}, & k_{\mathrm{t}}^{\prime}=\frac{E a^{3} b}{12 l} ; \\ k_{1}=\frac{E b t^{0.5}}{\pi\left(r^{0.5}-0.5 t^{0.5}\right)}, & k_{\mathrm{l}}^{\prime}=\frac{E a b}{l} ; \\ k_{\mathrm{b}}=\frac{2 E b t^{2.5}}{3 \pi r^{1.5}(3 r+t)}, & M_{\mathrm{t}}=k_{\mathrm{t}} \Delta \theta ;\end{cases}
$$

where $E$ is the elastic modulus, $b$ is the thickness of the flexure hinge, $r$ and $t$ are radius and smallest thickness of the circular notched hinge, $a$ and $l$ are the width and length of the prismatic beam, respectively.

Supposing that an input displacement $\delta_{\text {in }}$ is applied at the input-end of the mechanism, the corresponding input force and output displacement can be expressed by 


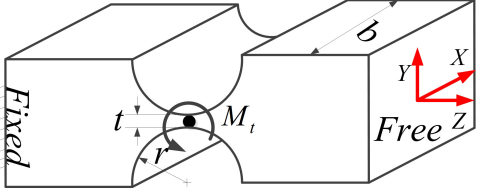

(a)

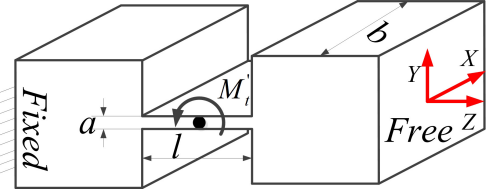

(b)

Figure 6. The flexure hinges: (a) the circular notched hinge; (b) the prismatic beam.

$\delta_{\text {out }}=\lambda_{\mathrm{AR}} \delta_{\text {in }}$

$F_{\text {in }}=K_{\text {in }} \delta_{\text {in }}$

where $\lambda_{\mathrm{AR}}$ and $K_{\text {in }}$ are the amplification ratio and input stiffness of the micromanipulator, respectively.

Due to the symmetry, only half of the amplifier mechanism is analyzed. According to the assumptions of literatures (Su and Yang, 2001a), force analysis simplified diagrams of the mechanical amplifiers are drawn up as shown in Fig. 7, where the Fig. $7 b$ and $c$ are two kinds of different one-stage amplifiers (Su and Yang, 2001b). Therefore, in consideration of the force and moment balance at the equilibrium state of the beam 2 , the following equations can be derived as

$F_{O_{3} y}=F_{O_{2} y}+F_{B_{y}}$

$F_{O_{3} y} l_{3}=F_{B_{y}} l_{4}+2 M_{\mathrm{t}}+M_{\mathrm{t}}^{\prime}$

where $M_{\mathrm{t}}$ and $M_{\mathrm{t}}{ }^{\prime}$ are bending moments of the points $O_{2}$ and $B$. Additionally, the forces $F_{O_{2} y}$ and $F_{B_{y}}$ can be calculated through multiplying stiffness by displacement. They are expressed by the following formulas

$F_{O_{2} y}=k_{O_{2} y} \delta_{2}, F_{B_{y}}=k_{B_{1}}\left(l_{4} \theta_{2}+\delta_{2}\right)$

$M_{\mathrm{t}}=k_{\mathrm{O}_{2} \mathrm{t}} \theta_{2}, M_{\mathrm{t}}^{\prime}=k_{B_{\mathrm{t}}} \theta_{2}$

where $k_{O_{2} y}$ and $k_{B_{1}}$ are the lateral bending stiffness caused by force $F_{O_{2} y}$ and output stiffness of the point $B$, respectively. $k_{O_{2} \mathrm{t}}$ and $k_{B_{\mathrm{t}}}$ are the rotational stiffness of the points $\mathrm{O}_{2}$ and $B$.

Substituting Eqs. (7) and (8) into Eqs. (5) and (6), the $\theta_{2}$ and $\delta_{2}$ can be derived as

$\theta_{2}=\frac{\left(k_{O_{2} y}+k_{B_{1}}\right) l_{3}-k_{B_{1}} l_{4}}{\left(k_{O_{2} y}+k_{B_{1}}\right)\left(k_{O_{2} \mathrm{t}}+k_{B_{\mathrm{t}}}\right)+2 k_{O_{2} y} k_{B_{1}} l_{4}^{2}} F_{O_{3} y}$
$\delta_{2}=\frac{k_{O_{2} \mathrm{t}}+k_{B_{\mathrm{t}}}+k_{B_{1}} l_{4}^{2}-k_{B_{1}} l_{3} l_{4}}{\left(k_{O_{2} y}+k_{B_{1}}\right)\left(k_{O_{2} \mathrm{t}}+k_{B_{\mathrm{t}}}\right)+2 k_{O_{2} y} k_{B_{1}} l_{4}^{2}} F_{O_{3} y}$

Therefore, the amplification ratio and input stiffness of the beam 2 can be calculated by the following equations

$$
\begin{aligned}
& \lambda_{2}=\frac{l_{4} \theta_{2}+\delta_{2}}{l_{3} \theta_{2}+\delta_{2}} \\
& k_{\text {in } 2}=\frac{F_{O_{3} y}}{l_{3} \theta_{2}+\delta_{2}}
\end{aligned}
$$

Then, substituting Eqs. (9) and (10) into Eqs. (11) and (12), following formulas can be obtained as

$$
\begin{aligned}
& \lambda_{2}=\frac{k_{O_{2} y} l_{3} l_{4}+2 k_{O_{2} \mathrm{t}}+k_{B_{\mathrm{t}}}}{k_{O_{2} y} l_{3}^{2}+2 k_{O_{2} \mathrm{t}}+k_{B_{\mathrm{t}}}+k_{B_{1}}\left(l_{4}-l_{3}\right)^{2}} \\
& k_{\mathrm{in} 2}=\frac{\left(k_{O_{2} y}+k_{B_{1}}\right)\left(2 k_{O_{2} \mathrm{t}}+k_{B_{\mathrm{t}}}\right)+k_{O_{2} y} k_{B_{1}} l_{4}^{2}}{k_{O_{2} y} l_{3}^{2}+2 k_{O_{2} \mathrm{t}}+k_{B_{\mathrm{t}}}+k_{B_{1}}\left(l_{4}-l_{3}\right)^{2}}
\end{aligned}
$$

Similarly, considering the relationship of force and moment balance at the equilibrium state for the beam 1 as shown in Fig. 7b, the following formulas can be obtained by

$$
\begin{aligned}
& F_{O_{3} y}+F_{A_{y}}=k_{O_{1} 1} \delta_{1} \\
& F_{A_{y}} l_{1}=F_{O_{3} y} l_{2}+k_{O_{3} \mathrm{t}} \theta_{1}+k_{O_{1} \mathrm{t}} \theta_{1} \\
& F_{O_{3} y}=k_{2}\left(l_{2} \theta_{1}-\delta_{1}\right)
\end{aligned}
$$

where $k_{O_{1} 1}$ and $k_{O_{1}}$ are axial stiffness and rotational stiffness of the point $O_{1} ; k_{O_{3} t}$ is rotational stiffness; $k_{2}$ is proposed as the stiffness of the series connection between the flexure hinge $\mathrm{O}_{3}$ and the beam 2, which can be expressed by

$k_{2}=\frac{k_{\mathrm{in} 2} k_{O_{3} 1}}{k_{O_{3} 1}+k_{\mathrm{in} 2}}$

where $k_{O_{3} 1}$ is axial stiffness of the flexure hinge $O_{3}$. Combining the Eq. (15) into Eq. (18), the $\theta_{1}$ and $\delta_{1}$ can be calculated by

$$
\begin{aligned}
& \theta_{1}=\frac{k_{O_{1} 1} l_{1}+k_{2}\left(l_{1}+l_{2}\right)}{\left(k_{O_{3} \mathrm{t}}+k_{O_{1} \mathrm{t}}\right)\left(k_{O_{1} 1}+k_{2}\right)+k_{O_{1} 1} k_{2} l_{2}^{2}} F_{A_{y}} \\
& \delta_{1}=\frac{k_{O_{3} \mathrm{t}}+k_{O_{1} \mathrm{t}}+k_{2} l_{2}^{2}+k_{2} l_{1} l_{2}}{\left(k_{O_{3} \mathrm{t}}+k_{O_{1} \mathrm{t}}\right)\left(k_{O_{1} 1}+k_{2}\right)+k_{O_{1} 1} k_{2} l_{2}^{2}} F_{A_{y}}
\end{aligned}
$$

Therefore, according to the Fig. 7b, amplification ratio and input stiffness of the beam 1 can be represented by

$$
\begin{gathered}
\lambda_{1}=\frac{l_{2} \theta_{1}-\delta_{1}}{l_{1} \theta_{1}+\delta_{1}} \\
k_{\mathrm{in} 1}=\frac{F_{A_{y}}}{l_{1} \theta_{1}+\delta_{1}}
\end{gathered}
$$

Substituting Eqs. (19) and (20) into Eqs. (21) and (22), they can be rewritten by

$\lambda_{1}=\frac{k_{O_{1} 1} l_{1} l_{2}-\left(k_{O_{3} \mathrm{t}}+k_{O_{1} \mathrm{t}}\right)}{k_{O_{1} 1} l_{1}^{2}+k_{O_{1} \mathrm{t}}+k_{O_{3} \mathrm{t}}+k_{2}\left(l_{1}+l_{2}\right)^{2}}$ 


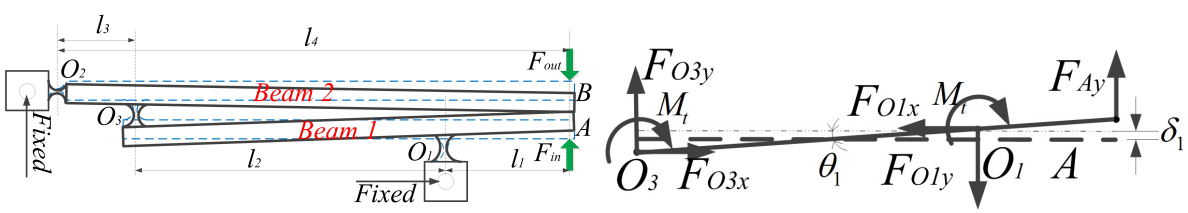

(a)

(b)

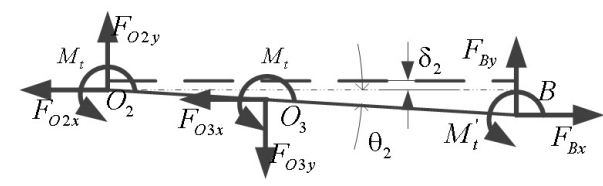

(c)

Figure 7. Force diagram: (a) the deformation of the half of the amplifier; (b) forced diagram of beam 1; (c) Forced diagram of beam 2.

$k_{\text {in } 1}=\frac{\left(k_{O_{1} 1}+k_{2}\right)\left(k_{O_{3} \mathrm{t}}+k_{O_{1} \mathrm{t}}\right)+k_{O_{1} 1} k_{2} l_{2}^{2}}{k_{O_{1} 1} l_{1}^{2}+k_{O_{1} \mathrm{t}}+k_{O_{3} \mathrm{t}}+k_{2}\left(l_{1}+l_{2}\right)^{2}}$

Consequently, the total amplification ratio of the two-stage lever displacement mechanism is calculated by

$\lambda_{\mathrm{AR}}=\lambda_{1} \lambda_{2}$

Additionally, total input force can be obtained by $F_{\text {in }}=$ $2 F_{A_{y}}$, since the amplifier is symmetric and is connected in parallel. Therefore, input stiffness can also be calculated by

$k_{\text {in }}=\frac{F_{\text {in }}}{l_{1} \theta_{1}+\delta_{1}}=\frac{2 F_{A_{y}}}{l_{1} \theta_{1}+\delta_{1}}=2 k_{\text {in } 1}$

For calculating the values of $k_{\text {in }}$ and $\lambda_{\mathrm{AR}}$, the stiffness $\left(k_{B_{1}}\right)$ of the output point $B$ must be firstly computed. According to the Fig. 4 , it can be observed that the output stiffness of the micromanipulator is the stiffness of components except from the driven amplifier. Simplified diagrams of output components are shown in Fig. 8 when the platform is driven in $y$ directions. The marked signs, $k_{1}{ }^{\prime}$ and $k_{\mathrm{t}}{ }^{\prime}$, are axial stiffness and rotational stiffness of the prismatic beam, respectively. And $k_{1}$ is the input stiffness of the two-stage amplifier when the input-end is at the point $B$ and output-end is at the point $A$ as shown in Fig. 7a.

Output displacement of the mechanism, $d_{\text {out }}$, can be obtained when an input displacement $d_{\text {in }}$ is applied at $y$ direction, whereas the amplifier of the $x$-direction will still hold since they have large transverse stiffness (Tang and $\mathrm{Li}, 2014)$. In addition, all rotational angles of the prismatic beams, $(\theta)$, are the same due to the identical structure. The potential energy stored in the flexible hinges can be derived as follows:

$$
\begin{aligned}
P_{\text {out }} & =\frac{1}{2} k_{N} d_{\text {out }}^{2} \\
& =4 \times \frac{1}{2} k_{\mathrm{t}}{ }^{\prime}(\theta)^{2}+2 \times \frac{1}{2} k_{\mathrm{t}}\left(\alpha_{1}^{2}+2 \beta_{1}^{2}+\gamma_{1}^{2}\right)
\end{aligned}
$$

where $\alpha_{1}, \beta_{1}$ and $\gamma_{1}$ are rotational angles of flexible hinges of the amplifier and can be written by

$\theta=\left(\frac{d_{\text {out }}}{l_{5}}\right)^{2}, \alpha_{1}=\left(\frac{d_{\text {out }}}{l_{4}}\right)^{2} ;$ $\beta_{1}=\left(\frac{d_{\mathrm{out}}}{3 l_{2}}\right)^{2}, \gamma_{1}=\left(\frac{d_{\mathrm{out}}}{5 l_{1}}\right)^{2}$;

As shown in Fig. 8b, the stiffness $k_{N}$ can be achieved by

$k_{N}=\frac{4 k_{\mathrm{t}}{ }^{\prime}}{l_{5}^{2}}+2 k_{\mathrm{t}}\left(\frac{1}{l_{4}^{2}}+\frac{2}{9 l_{2}^{2}}+\frac{1}{25 l_{1}^{2}}\right)$

According to the series-parallel relationship of the mechanism, the output stiffness $k_{\text {out }}$ can be expressed by the following formula

$$
\begin{aligned}
k_{\mathrm{out}} & =\frac{k_{N} k_{a}}{k_{N}+k_{a}} \\
& =\frac{4 k_{1}{ }^{\prime} k_{\mathrm{t}}{ }^{\prime}+2 k_{\mathrm{t}} k_{1}{ }^{\prime} l_{5}^{2} M}{k_{\mathrm{l}}{ }^{\prime} l_{5}^{2}+2 k_{\mathrm{t}}{ }^{\prime}+k_{\mathrm{t}} l_{5}^{2} M}
\end{aligned}
$$

where $M=\frac{1}{l_{4}^{2}}+\frac{2}{9 l_{2}^{2}}+\frac{1}{25 l_{1}^{2}}, k_{\mathrm{t}}{ }^{\prime}$ and $k_{1}{ }^{\prime}$ are rotational stiffness and axial stiffness of the prismatic beam.

Because circular notched hinges and prismatic beams used in this study are identical, thus their stiffness can be expressed by

$k_{O_{1} \mathrm{t}}=k_{O_{2} \mathrm{t}}=k_{O_{3} \mathrm{t}}=k_{A_{\mathrm{t}}}=k_{\mathrm{t}}$

$k_{O_{1} 1}=k_{O_{3} 1}=k_{1}$

$k_{O_{2} y}=k_{\mathrm{b}}$

$k_{B_{\mathrm{t}}}=k_{\mathrm{t}}{ }^{\prime}$

Due to the symmetry of the mechanism, the stiffness $k_{B_{1}}$ can be obtained by

$k_{B_{1}}=0.5 k_{\text {out }}$

Combining the Eqs. (25) and (32), at the same time considering Eq. (2), total amplification ratio and input stiffness of the mechanism, which can be represented by the designed parameters including $r, t, a, b$ and $l_{i}(i=1, \ldots, 5)$, are not provided here due to page limitations. To conveniently calculate the corresponding calculations, geometric parameters and properties of the material (AL7075-T6) of mechanism are listed in Table 1. 


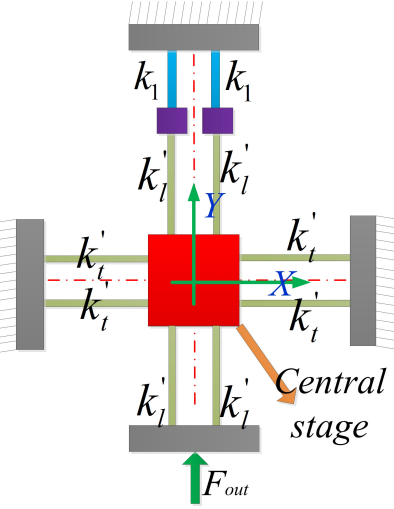

(a)

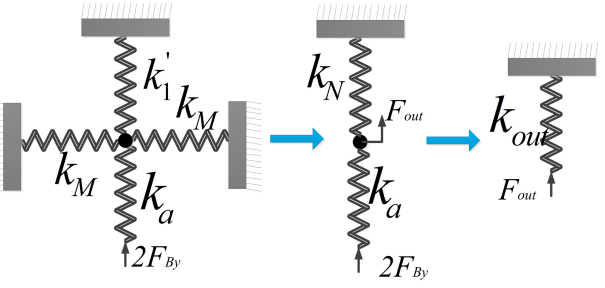

(b)

Figure 8. The stiffness of output mechanism: (a) the diagram of the output mechanism; (b) the output stiffness transformation diagram.

Table 1. Geometric parameters $(\mathrm{mm})$ and properties of material of the mechanism.

\begin{tabular}{|c|c|c|c|c|c|c|c|c|}
\hline$r$ & $t$ & $a$ & $b$ & $l_{1}$ & $l_{2}$ & $l_{3}$ & $l_{4}$ & $l_{5}$ \\
\hline 1.5 & 0.5 & 0.5 & 10 & 7.5 & 12.5 & 10 & 30 & 30 \\
\hline \multicolumn{2}{|c|}{$E$} & \multicolumn{2}{|c|}{$\varepsilon$} & \multicolumn{3}{|c|}{$\rho$} & \multicolumn{2}{|c|}{$\sigma_{y}$} \\
\hline \multicolumn{2}{|c|}{$71.7 \mathrm{GPa}$} & \multicolumn{2}{|c|}{0.33} & \multicolumn{3}{|c|}{$7810 \mathrm{~kg} \mathrm{~m}^{-3}$} & \multicolumn{2}{|c|}{$503 \mathrm{MPa}$} \\
\hline
\end{tabular}

\subsection{Reachable workspace analysis}

For a 2-DOF motion platform, supposing that the $\mathrm{AR}_{\mathrm{m}}$ is denoted as the amplification ratio of the mechanism and $S$ is looked as the stroke of the PZT actuator, the workspace of the micromanipulation stage is represented by $\mathrm{AR}_{\mathrm{m}} S \times \mathrm{AR}_{\mathrm{m}} S$. In this study, the bending stress only occurs on the cross section of flexible hinge when axial tension and shearing effects are not taken into consideration. Thus stress $\left(\sigma_{r}\right)$ can be calculated as the following equation:

$\max \left\{\sigma_{r}\right\} \leq \frac{\sigma_{y}}{s}$

where $\sigma_{y}$ and $s>1$ are the yield strength and the safety factor of the material. In addition, maximal stress $\sigma_{r}^{\max }$ occurs when the angular displacement $\theta_{\max }$ is maximum. It can be expressed using the following formula

$\sigma_{r}^{\max }=\frac{4 E r^{2} k_{\mathrm{c}}}{f(\beta) t^{2}} \theta_{\max }$

where $k_{\mathrm{c}}$ and $f(\beta)$ are the dimensionless concentration factor and compliance factor, respectively. They are expressed by (Smith, 2000).

$k_{\mathrm{c}}=(1+\beta)^{9 / 20}$

$$
f(\beta)=\frac{1}{2 \beta+\beta^{2}}\left[\begin{array}{l}
\frac{3+4 \beta+2 \beta^{2}}{(1+\beta)\left(2 \beta+\beta^{2}\right)}+ \\
\frac{6(1+\beta)}{\left(2 \beta+\beta^{2}\right)^{3 / 2}} \tan ^{-1}\left(\sqrt{\frac{2+\beta}{\beta}}\right)
\end{array}\right]
$$

where $\beta=t / 2 r$ is the dimensionless geometry factor.

Assuming that the maximal input displacement is $\delta_{\text {in }}^{\max }$, corresponding to the output displacement of the motion stage is $\lambda_{\mathrm{AR}} \delta_{\mathrm{in}}^{\max }$. According to the mechanical principle, the maximal stress may occur on the hinge $O_{3}$ connecting the two lever beams, since it not only suffers from the angular deformation created by output displacement, but may be also subjected to the angular deformation produced by the input displacement. Therefore, the maximal deformation angle can be derived by

$\theta_{\max }=\frac{\lambda_{\mathrm{AR}} \delta_{\mathrm{in}}^{\max }}{l_{4}}+\frac{\lambda_{1} \delta_{\mathrm{in}}^{\max }}{l_{2}}$

Substituting Eqs. (37) into (34) and considering the Eqs. (36) and (33) at the same time, the maximal input displacement can be calculated as follows

$\delta_{\text {in }}^{\max } \leq \frac{l_{2} l_{4} t^{2} f(\beta) \sigma_{y}}{4 E r^{2} k_{\mathrm{c}} s\left(l_{2} \lambda_{\mathrm{AR}}+l_{4} \lambda_{1}\right)}$

Substituting values of the Table 1 into the Eq. (38) and letting the safety factor be 1.78. Maximal input displacement can be calculated as follows

$\delta_{\text {in }}^{\max } \leq 49.2 \mu \mathrm{m}$

Considering the amplification ratio of the mechanism, the output displacement of the stage can be $236.2 \mu \mathrm{m}$. Due to the symmetry of the mechanism, so the reachable workspace of the platform can be expressed as $236.2 \mu \mathrm{m} \times 236.2 \mu \mathrm{m}$.

\subsection{Natural frequency analysis}

To analyze free vibration of the 2-DOF micro positioning stage, the natural frequencies are achieved by utilizing Lagrange's method. The coordinate vector $\eta=\left[\eta_{1}, \eta_{2}\right]^{T}$ is used 


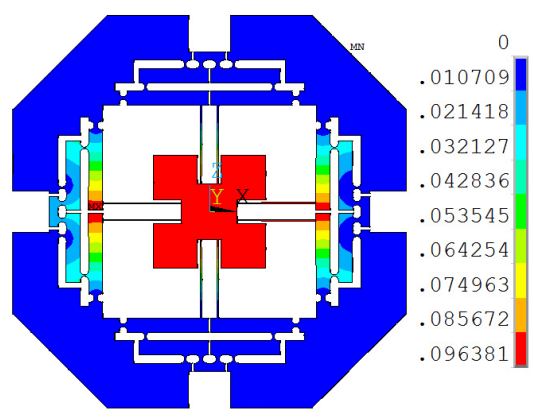

(a)

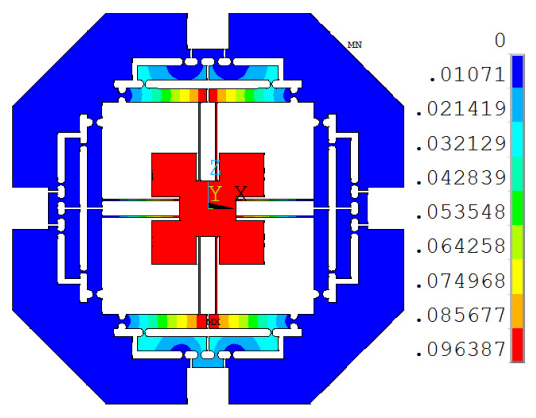

(b)

Figure 9. Deformations: (a) input at $x$ direction and (b) input at $y$ direction.

to describe the motions of $x$ - and $y$-axes. The kinetic energy $(T)$ and potential energy $(V)$ stored in the mechanism can be expressed by the selected generalized coordinate and their derivations (Li et al., 2013). And then, substituting the kinetic and potential energies into the following Lagrange's equation

$$
\frac{\mathrm{d}}{\mathrm{d} t} \cdot \frac{\partial T}{\partial \dot{\eta}_{i}}-\frac{\partial T}{\partial \eta_{i}}+\frac{\partial V}{\partial \eta_{i}}=F_{i}
$$

where $i=1,2$, corresponds to the free vibration of $x$ - and $y$-directions of the stage.

$\mathbf{M} \ddot{\eta}+\mathbf{K} \eta=\mathbf{0}$

where the equivalent mass $\mathbf{M}=\operatorname{diag}\{M\}$ and stiffness $\mathbf{K}=$ $\operatorname{diag}\{k\}$ are the $2 \times 2$ diagonal matrices, along with

$$
\begin{aligned}
M & =2 m_{0} \lambda_{\mathrm{AR}}^{2}+4 m_{1}+\frac{7}{3} m_{2}+\frac{7}{3} m_{3} \lambda_{1}^{2} \\
& +\frac{7}{3} m_{4} \lambda_{\mathrm{AR}}^{2}+\frac{16}{3} m_{5} \lambda_{\mathrm{AR}}^{2} \\
K & =k_{\text {in }}
\end{aligned}
$$

where $m_{i}(i=0,1, \ldots, 5)$ are shown in the Figs. 2 and 5.

Solving the Eq. (41), the natural frequency of the stage can be obtained as

$f=\frac{1}{2 \pi}\left(\frac{K}{M}\right)^{0.5}$

which has the unit of Hertz.

\section{Verification and evaluation with FEA}

In this section, the established models to evaluate the properties of the 2-DOF micromanipulation stage on aspects of amplification ratio, input stiffness, reachable workspace, and natural frequencies are verified by using FEA software (Li et al., 2012).

\subsection{Model verification}

For analyzing the amplification ratio of the proposed mechanism, an input displacement with $20 \mu \mathrm{m}$ is separately provided in the $x$ - and $y$-directions and the deformation of the mechanism is shown in Fig. 9a and b, respectively. Therefore, the corresponding output displacements can be measured by selecting the points of the surface of the central platform and the average values of them are calculated with 87.58 and $87.57 \mu \mathrm{m}$. Additionally, the force can be also measured with $43.20 \mathrm{~N}$. Therefore, the amplification ratio and input stiffness of the mechanism can be calculated as 4.38 and $2.16 N \mu \mathrm{m}^{-1}$, respectively.

Considering the workspace within the allowable maximum stress, the maximal input displacement of the Eq. (39) is simultaneously applied at the $x$ - and $y$-directions. Thus, the maximal stress distribution and the corresponding displacement deformations are shown in Fig. 10. It can be seen that the maximal stress is $278.1 \mathrm{MPa}$ at the point $\mathrm{O}_{3}$, which is less than the allowable stress of the material. The Fig. 10b, $\mathrm{c}$ and $\mathrm{d}$ indicate the maximal output displacements of the whole mechanism. Based on the aforementioned analysis, the reachable workspace of the mechanism is measured with $215.50 \mu \mathrm{m} \times 215.50 \mu \mathrm{m}$.

In order to analyze parasitic motion of the mechanism, the maximal input displacement is separately applied to the $x$ and $y$-axes. As shown in Fig. 11a and b, which is the output displacement of $x$-direction with $215.50 \mu \mathrm{m}$ and parasitic motion at $y$-direction with $0.034 \mu \mathrm{m}$, respectively. Similarly, the Fig. 11c and d are the output displacement at $y$-direction with $215.50 \mu \mathrm{m}$ and parasitic motion of $x$-direction with $0.036 \mu \mathrm{m}$. The parasitic motions of the $x$ - and $y$-directions may be caused by the model errors and the deformations of the prismatic joint.

For verifying the dynamic model with the natural frequencies, the first four modal shapes of the structure are expressed in Fig. 12. The first modal shape is the rotational motion, which has the frequency with $179.99 \mathrm{~Hz}$. The second and third modal shapes in the $x$-and $y$-directions are almost the 


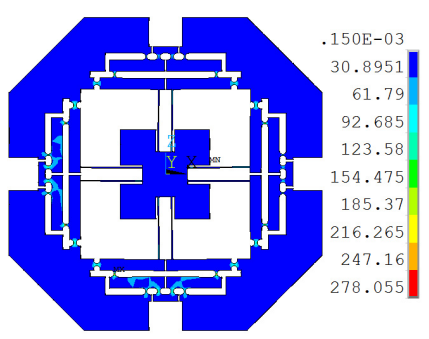

(a)

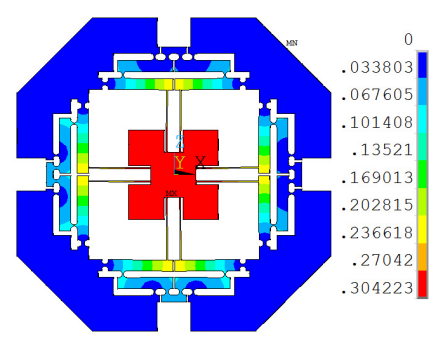

(b)

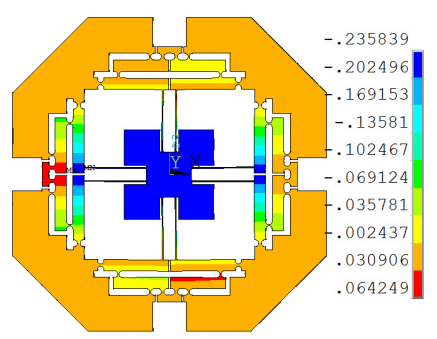

(c)

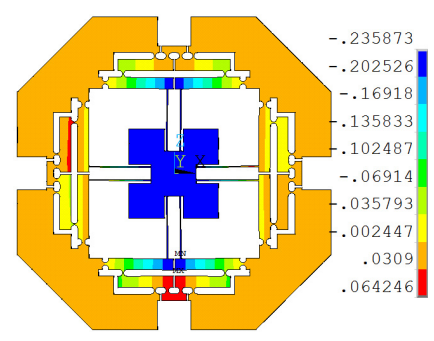

(d)

Figure 10. The stress distribution and deformation diagrams: (a) maximal stress; (b) total output displacement; (c) output displacement at $x$-direction; (d) output displacement at $y$-direction.

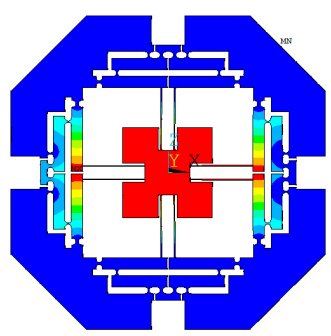

(a)
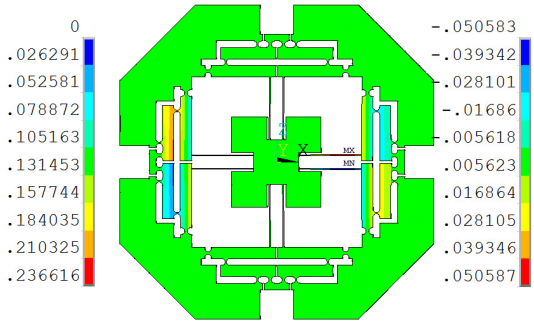

(b)

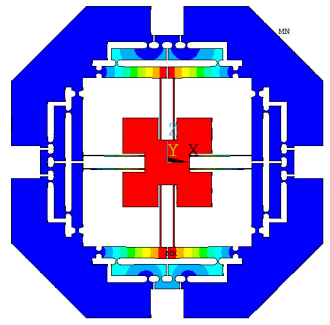

(c)
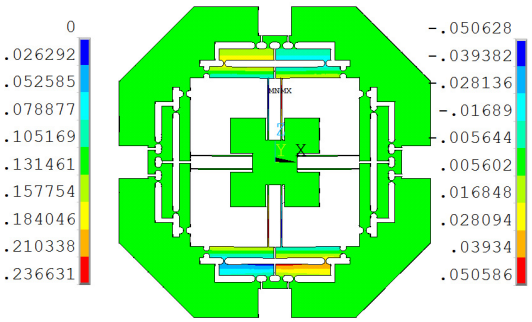

(d)

Figure 11. Output displacements and parasitic motions: (a) output displacement at $x$-axis; (b) parasitic motion at $y$-direction; (c) output displacement at $y$-axis; (d) parasitic motion at $x$-direction.

Table 2. Comparison results between calculated values and simulated values.

\begin{tabular}{lrrr}
\hline & Calculated & FEM & Deviation \\
\hline$\lambda_{\mathrm{AR}}$ & 4.81 & 4.38 & $9.82 \%$ \\
$k_{\text {in }}$ & $2.08 \mathrm{~N} \mathrm{\mu m}^{-1}$ & $2.16 \mathrm{~N} \mathrm{\mu m}^{-1}$ & $3.70 \%$ \\
$f$ & $341.25 \mathrm{~Hz}$ & $354.21 \mathrm{~Hz}$ & $3.66 \%$ \\
$\delta_{\text {out }}$ & $236.7 \mu \mathrm{m}$ & $215.50 \mu \mathrm{m}$ & $9.84 \%$ \\
$\sigma_{\text {max }}$ & $282.6 \mathrm{MPa}$ & $278.1 \mathrm{MPa}$ & $1.62 \%$ \\
\hline
\end{tabular}

same with values of 354.21 and $355.32 \mathrm{~Hz}$, respectively. The fourth modal shape of the $Z$-direction is about $867.23 \mathrm{~Hz}$.

\subsection{Discussions}

For further indicating the rationality of the design ( $\mathrm{Li}$ and $\mathrm{Wu}, 2016$ ), a comparison between the calculated values and the simulated values of FEA, in terms of amplification ratio, input stiffness, natural frequency, and maximum output displacement, is listed in Table 2. It can be observed that the maximal error is the output displacement and amplification ratio by using the benchmark of FEA. The reasons may be that the transverse deformation caused by the transverse force as shown in Fig. 7 is not taken into account in the calculated models and flexure hinge itself. In addition, the maximal stress is under the truly calculated value. The natural
Table 3. Cross coupling in two axes.

\begin{tabular}{lrrr}
\hline & $x$ input & $y$ input & $x y$ inputs \\
\hline$x$ output & $215.50 \mu \mathrm{m}$ & $0.036 \mu \mathrm{m}$ & $214.99 \mu \mathrm{m}$ \\
$y$ output & $0.034 \mu \mathrm{m}$ & $215.50 \mu \mathrm{m}$ & $215.01 \mu \mathrm{m}$ \\
Cross coupling & $0.1 \%$ & $0.1 \%$ & $/$ \\
\hline
\end{tabular}

frequencies of the $x$ - and $y$-directions are almost equivalent, which demonstrate that their performances are identical.

Cross coupling is the key performance of the 2-DOF micro-positioning stage. As shown in Fig. 11, the results of the maximum output displacements in two axes are listed in the Table 3. The output displacement of $x$-axis is $215.50 \mu \mathrm{m}$ when input displacement is separately applied at $x$-direction, while the parasitic displacement of $y$-direction caused by $x$-direction is only $0.034 \mu \mathrm{m}$. Taking input displacement as the benchmark, coupling error of the $y$-axis is only for $0.1 \%$. Similarly, the parasitic motion of $x$-direction caused by $y$-direction is $0.036 \mu \mathrm{m}$ with the output displacement is $215.50 \mu \mathrm{m}$ at $y$-direction. Thus, coupling error on the $x$-axis is only for $0.1 \%$ more than that of $y$-axis. Additionally, output displacements of $x$ - and $y$-axes are up to 214.99 and $215.01 \mu \mathrm{m}$ when the input displacements are simultaneously applied in the $x$ - and $y$-directions. Based on the aforementioned analysis, the 2-DOF micromanipulation stage has low cross coupling and owns the best decoupling property. 


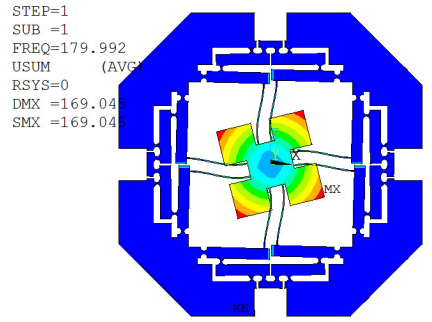

(a)

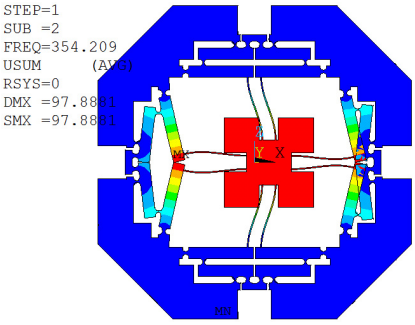

(b)

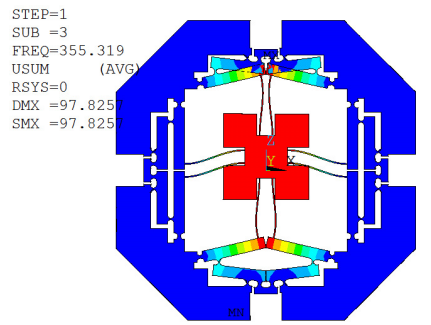

(c)

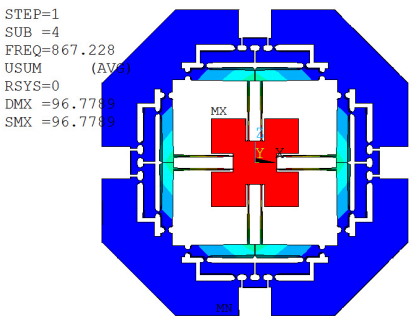

(d)

Figure 12. The first four modal shapes of the mechanism: (a) the first modal shape; (b) the second modal shapes; (c) the third modal shape; (d) the fourth modal shape.

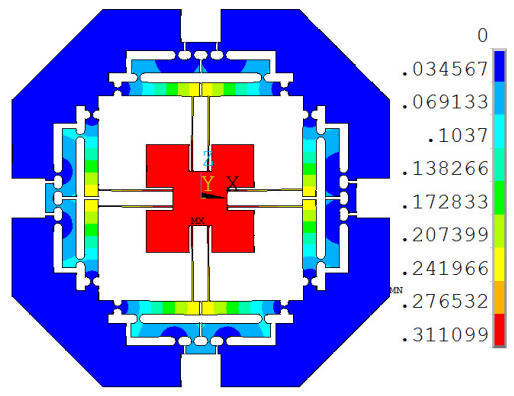

(a)

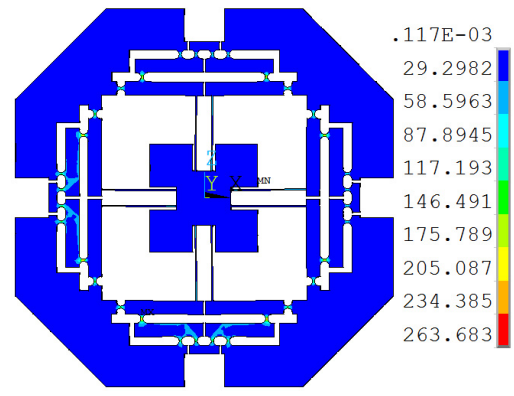

(b)

Figure 13. The verification of FEA: (a) deformation result; (b) stress distribution.

\section{Structure optimization}

The parameters of the architecture by using the Genetic Algorithm (GA) method are optimized to obtain the best kinematic characteristic of the $x y$ stage (Wang et al., 2014). In practical applications, the actual amplification ratio of mechanical amplifier is less than the theoretical value, which is arisen from the combination of the deformation of hinge and lever arm bending. Additionally, considering analytical models overestimate the performance of the stage with deviations around $5 \%-30 \%$, a compensation factor, $\varepsilon=0.75$, is used in the optimization process to rebuild the models.

\subsection{Optimization problem description}

Based on aforementioned equations, which reveal that the performances of the 2-DOF micro-positioning stage rely on the relative parameters. All established models mainly include these parametric variables: $r, t, a, b, l_{1}, l_{2}, l_{3}, l_{4}, l_{5}$, which influence the synthetical property of the stage.

For the mechanism with a special thickness $(b=10 \mathrm{~mm}$ in this study), only eight parameters $\left(r, t, a, l_{1}, l_{2}, l_{3}, l_{4}, l_{5}\right)$ need to be optimized. While other parameters can be determined by taking into account the length and width restrictions of the PZT with the addition of an appropriate assembling space. Otherwise, input stiffness of the mechanism should not surpass the minimum stiffness of the PZT. According to the ge- ometrical relation of the structure, these variables should satisfy the following equation

$$
\begin{aligned}
& 2 r+t \leq l_{1} \leq l_{2} \\
& 2.5+r \leq l_{3} \leq l_{4} \\
& 2 l_{4}+2 r \leq 2 l_{5}+14 \\
& l_{4}-l_{3}=l_{1}+l_{2}
\end{aligned}
$$

With the selection of the amplification ratio of the stage as an objective function, the optimization process can be stated as follows:

a. Maximum: Amplification ratio $\left(\lambda_{\mathrm{AR}} \geq 7\right)$.

b. Variables to be optimized: $r, t, a, l_{1}, l_{2}, l_{3}, l_{4}$, and $l_{5}$.

c. Subjecting to:

1. Input stiffness value $\varepsilon k_{\text {in }} \leq K_{\mathrm{PZT}}$;

2. Natural frequency $\varepsilon f \geq 150 \mathrm{~Hz}$;

3. Theoretical amplification ratio $\mathrm{AR} \geq 7$;

4. Suffering from the restrictions of Eq. (45);

5. The ranges of parameters:

$1.5 \mathrm{~mm} \leq r \leq 2.0 \mathrm{~mm}, 0.3 \mathrm{~mm} \leq t \leq 0.8 \mathrm{~mm}$, $0.3 \mathrm{~mm} \leq a \leq 1 \mathrm{~mm}, 5 \mathrm{~mm} \leq l_{1} \leq 15 \mathrm{~mm}$, $7 \mathrm{~mm} \leq l_{2} \leq 20 \mathrm{~mm}, 5 \mathrm{~mm} \leq l_{3} \leq 15 \mathrm{~mm}$, $20 \mathrm{~mm} \leq l_{4} \leq 40 \mathrm{~mm}, 28 \mathrm{~mm} \leq l_{5} \leq 40 \mathrm{~mm}$. 


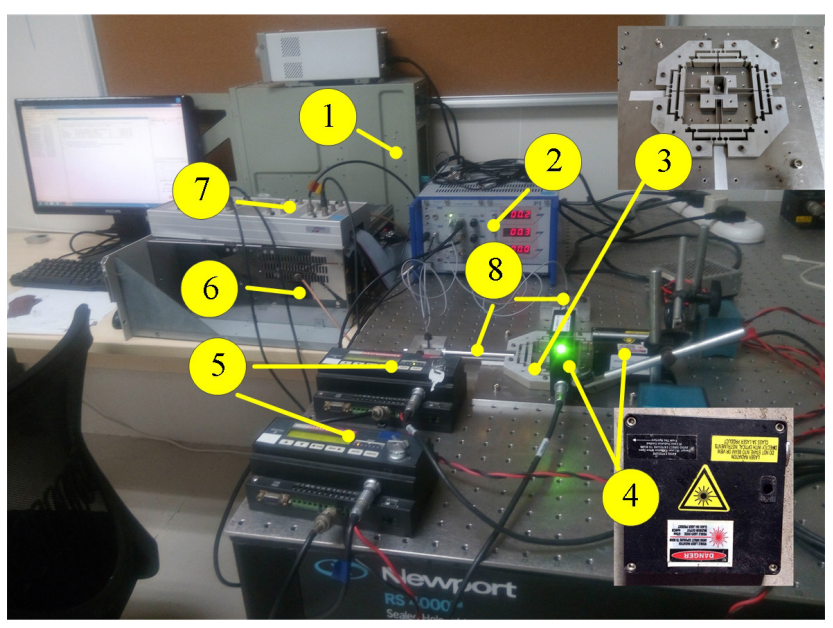

Figure 14. The experimental setup. (1) Host computer, (2) signal amplifier and controller, (3) compliant mechanism, (4) laser sensors, (5) laser collectors, (6) dSPACE controlling system, (7) DAQ board, (8) PZT actuators.

\subsection{Optimal results}

The Genetic Algorithm (GA) is adopted in the current issue due to its superiority of fast convergence, fewer calculating time and higher robustness over other method such as simulated annealing algorithm (Mccall, 2005). The optimization process is implemented by the GA toolbox of the MATLAB software and the results of optimized parameters are: $r=1.5 \mathrm{~mm}, t=0.78 \mathrm{~mm}, a=0.5 \mathrm{~mm}, l_{1}=5.47 \mathrm{~mm}$, $l_{2}=14.57 \mathrm{~mm}, l_{3}=7.90 \mathrm{~mm}, l_{4}=27.94 \mathrm{~mm}, l_{5}=28 \mathrm{~mm}$, which will lead to an $x y$ stage with $\lambda_{\mathrm{AR}}=7.87, k_{\text {in }}=$ $13.39 N \mu \mathrm{m}^{-1}$ and the natural frequency $f=367.07 \mathrm{~Hz}$.

After optimization, simulation is carried out for demonstrating performances of the optimized micromanipulation stage. Maximum input displacements are applied at $x$ - and $y$-axes and the total deflection and stress distribution of the mechanism are expressed in Fig. 13. It is observed that the output displacement of $x$-axis is $94.90 \mu \mathrm{m}$, and the corresponding input force is $214.63 \mathrm{~N}$. Therefore, the optimized mechanism has an amplification ratio of 4.75 with an input stiffness of $10.63 \mathrm{~N} \mathrm{\mu m}^{-1}$. In addition, maximal stress is $263.68 \mathrm{MPa}$, which is far less than the yield strength of the material.

Furthermore, the first four natural frequencies are also analyzed and their values are 174.26, 348.31, 350.14 and $850.65 \mathrm{~Hz}$, respectively. They are all less than the frequencies before optimizing and the frequencies of $x$ - and $y$-directions are very close to the calculated values, which demonstrate that the structure optimization is effective.

\section{Experimental tests}

\subsection{Experimental setup}

After fabricating, experimental setup of the micropositioning stage is shown in Fig. 14. Two PZTs with stroke of $90 \mu \mathrm{m}$ (model P-840.60 produced by Physik Instrumente, Inc.) are adopted to drive the micromanipulation stage, and the PZTs are actuated with a voltage of $0-100 \mathrm{~V}$ through a three-axis piezo-amplifier and driver (E-509 from the PI). Two laser displacement sensors and collectors (Microtrak II, head model: LTC-025-02, from MTI Instrument, Inc.) are used to measure the end-effector displacements of the two axes. The analog outputs of two sensors are connected to a PCI-based data acquisition (DAQ) board (PCI-6143 with 16-bit A/D convertors, from NI Corp.) through a shielded I/O connector block (SCB-68 from NI) with noise rejections. The digital outputs of the DAQ board are read by a host computer simultaneously.

Since the sensitivity of the laser sensor is $2.0 \mathrm{~mm} / 10 \mathrm{~V}$ and the maximum value of 16-bit digital signal corresponds to $10 \mathrm{~V}$, the resolution of the displacement detecting system can be calculated as

$\frac{2.0 \mathrm{~mm}}{10 \mathrm{~V}} \times \frac{10 \mathrm{~V}}{2^{16}}=0.031 \mu \mathrm{m}$

However, due to a considerable level of the noise, the resolution of the sensor is claimed $\pm 0.2 \mu \mathrm{m}$ by the manufacturer. In the process of practical precision motion control experiments, it is found that the limitation of the fabricated prototype mainly arises from the laser displacement sensors which have a not-high resolution of $\pm 0.2 \mu \mathrm{m}$.

\subsection{Open test}

To describe the dynamic properties of the proposed mechanism, the corresponding open-loop tests are carried out by using the dSPACE real-time simulation control system. As shown in Fig. 15a and b, which demonstrate respectively the output displacements and parasitic motions of $x$ - and $y$ directions when the input voltage is $u=30 \sin \left(\frac{\pi}{4} t-\frac{\pi}{2}\right)+30$. The results indicate that the actual amplification ratio of the mechanical amplifier in $x$-direction by comparing the input and output displacements is about 3.51 and the parasitic motion for the $y$-direction is about $0.32 \mu \mathrm{m}$. While one of $y$ direction is calculated as 3.43 and the parasitic motion for the $x$-direction is only $0.31 \mu \mathrm{m}$. Based on the aforementioned tests, the experimental results are less than the analytical results. The main reasons may come from the assembly errors of the system and the preloaded force of the PZT. Additionally, parasitic motions of the $x$ - and $y$-axes are very small, which can be ignored. Therefore, testing results demonstrate that the designed mechanism has excellent decoupled performance.

To further illustrate the kinematic performance of the micro-positioning stage, the testing results of the $x$ - and $y$ - 

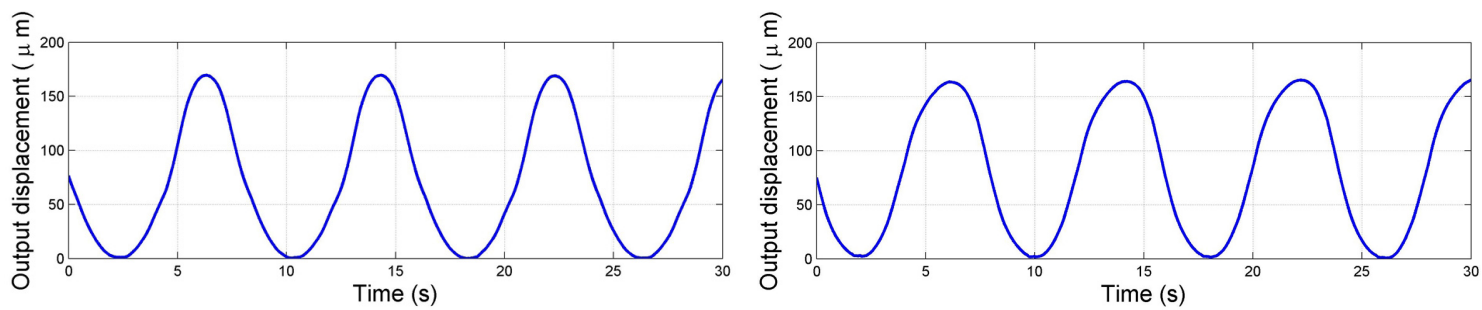

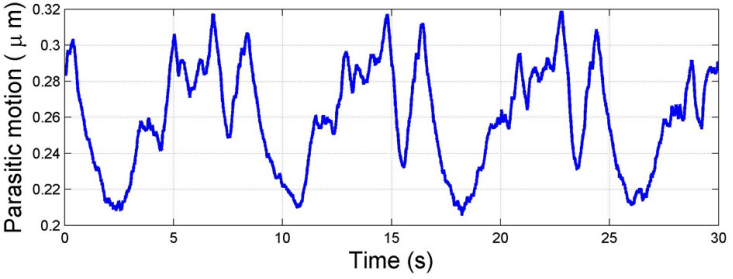

(a)

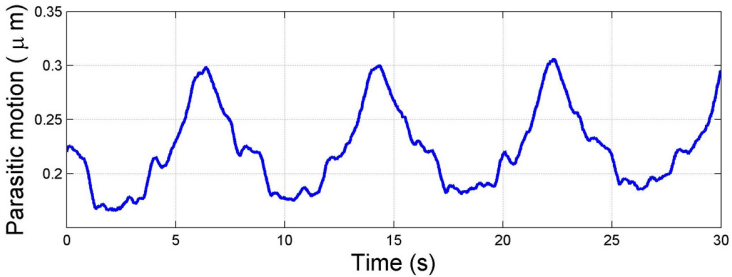

(b)

Figure 15. The output displacements and parasitic motions: (a) the output displacement of $x$-axis and parasitic motion of $y$-axis; (b) the output displacement of $y$-axis and parasitic motion of $x$-axis.

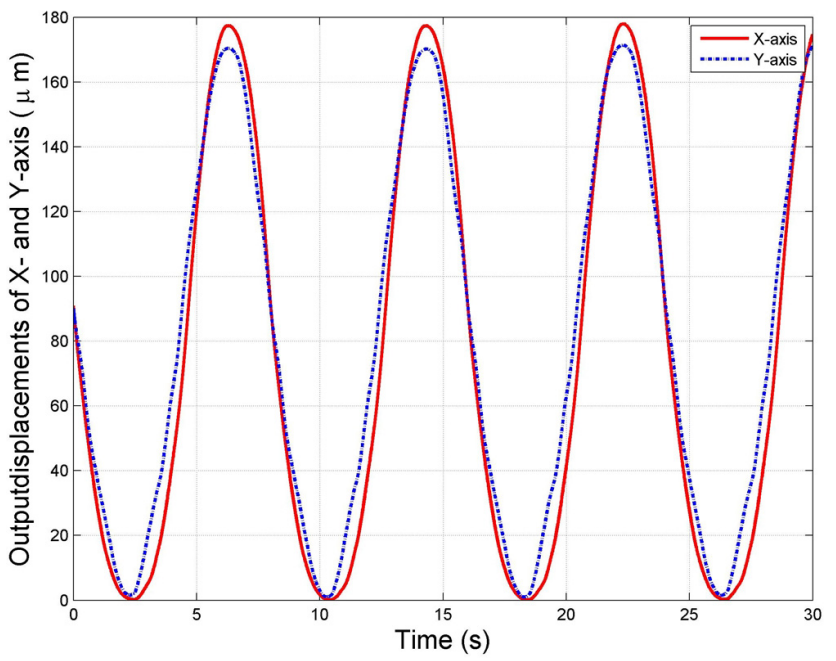

Figure 16. The output displacements of $x$ - and $y$-axes.

axes are shown in Fig. 16 when the two PZTs at $x$ - and $y$ directions are driven simultaneously. The results indicate that the output displacements of $x$ - and $y$-directions are not identical. The errors may be created by the preloaded force of PZTs and manufacturing defects between two axes.

\subsection{Tracking experiments}

For further validating the performances of micro/nanopositioning platform, a robust tracking controller is used to obtain the well tracking effect. The reference input displacement is the signal with different frequencies since the hysteresis phenomenon of piezoelectric actuator is a ratedependent hysteresis. Therefore, the robust controller can effectively eliminate the drawback caused by rate-dependent of
Table 4. Property comparisons for proposed $x y$ stages.

\begin{tabular}{lrrr}
\hline Refs. & $\begin{array}{r}\text { Frequency } \\
(\mathrm{Hz})\end{array}$ & $\begin{array}{r}\text { Cross-coupling } \\
(\%)\end{array}$ & $\begin{array}{r}\text { Workspace } \\
\left(\mu \mathrm{m}^{2}\right)\end{array}$ \\
\hline Ref. 3 & $2.7 k$ & $/$ & $25 \times 25$ \\
Ref. 5 & 831 & 2 & $119.7 \times 121.4$ \\
Ref. 9 & 720.52 & 5 & $19.2 \times 18.8$ \\
Ref. 11 & $2 k$ & 0.2 & $15 \times 15$ \\
Ref. 12 & 665.4 & 2 & $8 \times 8$ \\
This study & 348.31 & 0.1 & $169.6 \times 165.3$ \\
\hline
\end{tabular}

the PZT-actuated micromanipulator. The tracking results and corresponding errors is shown in Fig. 17, where output displacement is well tracking with the input displacement and the error is low than $0.01 \%$. Thus the optimal design is suitable for this compliant mechanism.

\subsection{Discussions}

From the Figs. 15 and 16, we can see that the output displacements of the $x$ - and $y$-axes are not exactly same, the main reasons maybe come from the errors coming from installation and manufacturing. Thus, the rotation of end-effector can exist, but it may be minor affections for the other orientation.

Based on aforementioned modeling, analyzing, and testing, the results demonstrate that the proposed 2-DOF micropositioning stage with mechanical amplifier owns some advantages, in terms of large motion, and low cross coupling. A comparison with other proposed 2-DOF stages are completed in Table 4. The natural frequencies of the presented stages in Ref. 3 and Ref. 11 are higher than other mechanisms, but their working ranges are very small, which seri- 


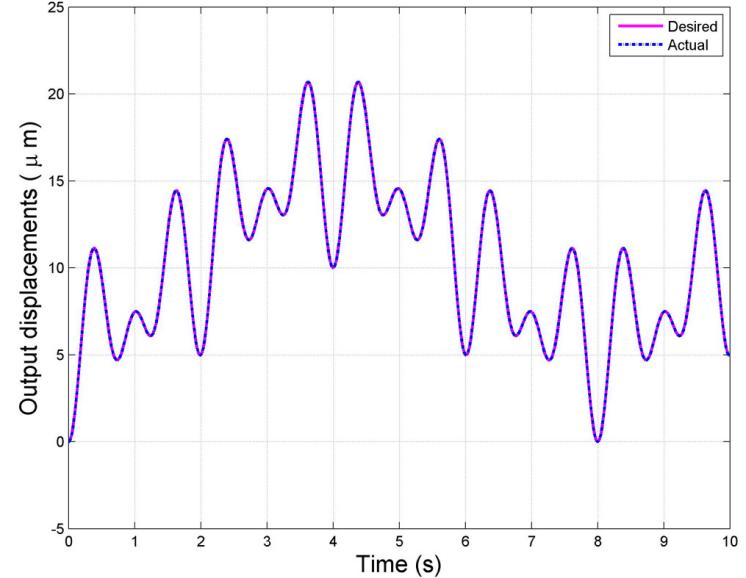

(a)

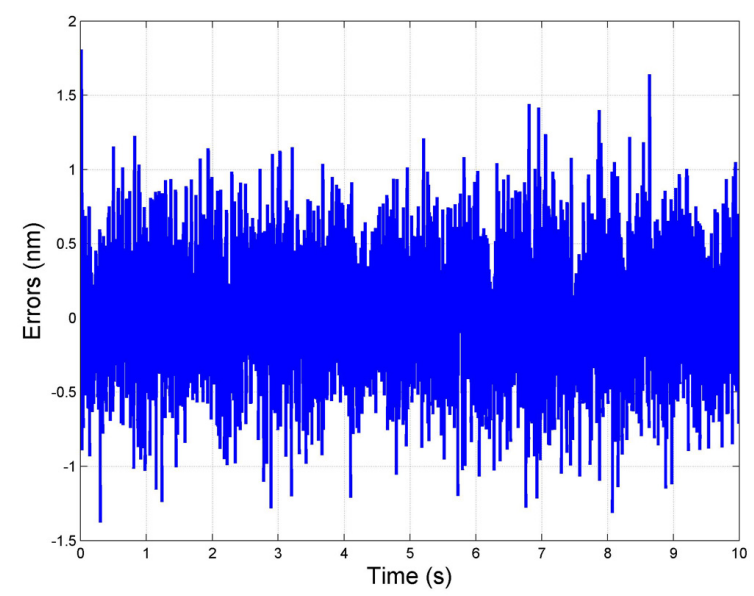

(b)

Figure 17. The tracking result and error: (a) the tracking result; (b) the tracking error.

ously limit their further applications. Additionally, the performances in terms of cross coupling and workspace of the other two stages presented in Ref. 5, Ref. 9 and Ref. 12 are obviously lower than the proposed in this study.

\section{Conclusions}

In this paper, a novel fully decoupled $x y$ micro-positioning stage with lever amplifier has been proposed. The designing process of the stage is provided in consideration of the decoupled property of the output motions. In addition, the analytical method and Lagrange's method are adopted for the kinematics and dynamics modeling of the mechanism with amplification ratio, stiffness, reachable workspace and natural frequency. The modeling verification and performance evaluation are carried out by FEA. Considering the performance requirement, a series of structural optimizations by using GA method have been implemented to improve the amplification ratio. Finally, prototype fabrication and experimental tests are implemented in detail. All results indicate that the maximal cross coupling of the 2-DOF micropositioning stage is less than $0.1 \%$ under the workspace for $169.6 \mu \mathrm{m} \times 165.3 \mu \mathrm{m}$ with the natural frequency of 348.31 .

For further study, intelligent controller is going to be considered to control the micro-positioning stage and precise position tracking will be carried out in our future work.

Data availability. This experimental data can be downloaded at https://pan.baidu.com/disk/home?errno=0\&errmsg=Auth Login Sucess \&\&bduss $=\&$ ssnerror $=0 \&$ traceid $=\# /$ all path $=/ \&$ vmode $=$ list (last access: 20 November 2018).
Author contributions. The main contribution for $\mathrm{ZW}$ includes the structural design, modeling analysis and the control. The contribution for co-author MH includes the structural optimization. And the contribution for co-author YL includes the structural design and the English writing error and grammar modification.

Competing interests. The authors declare that they have no conflict of interest.

Acknowledgements. This work was supported in part by Science and technology research project of department of education, Jiangxi, China (GJJ170568), National Natural Science Foundation of China (51575544, 51275353), Research Committee of The Hong Kong Polytechnic University (1-ZE97, G-YZ1G).

Edited by: Xichun Luo

Reviewed by: Calin-Octavian Miclosina and one anonymous referee

\section{References}

Choi, S. B., Han, S. S., Han, Y. M., and Thompson, B. S.: A magnification device for precision mechanisms featuring piezoactuators and flexure hinges: design and experimental validation, Mech. Mach. Theory, 42, 1184-1198, 2007.

Dong, J. D., Mukhopadhyay, D., and Ferreira, P. M.: Design, fabrication and testing of a silicon-on-insulator (SOI) MEMS parallel kinematics $X Y$ stage, J. Micromech. Microeng., 17, 1154-1161, 2007.

Dong, Y., Gao, F., and Yue, Y.: Modeling and experimental study of a novel 3-RPR parallel micro-manipulator, Robot. Cim.-Int Manuf., 37, 115-126, 2016.

Fan, S., Liu, H., and Fan, D.: Design and development of a novel monolithic compliant $X Y$ stage with centimeter travel range and high payload capacity, Mech. Sci., 9, 161-176, 2018. 
Hao, G., Li, H., and Kavanagh, R.: Design of Decoupled, Compact, and Monolithic Spatial Translational Compliant Parallel Manipulators Based on the Position Space Concept, P. I. Mech. Eng. C-J. Mec., 230, 367-378, 2016.

Koseki, Y., Tanikawa, T., Koyachi, N., and Arai, T.: Kinematic analysis of a translational 3-d.o.f. micro-parallel mechanism using the matrix method, Adv. Robotics, 16, 95-105, 2002.

Li, Y. M. and Wu, Z. G.: Design, analysis and simulation of a novel 3-DOF translational micromanipulator based on the PRB model, Mech. Mach. Theory, 100, 235-258, 2016.

Li, Y. M., Huang, J. M., and Tang, H.: A compliant parallel $X Y$ micromotion stage with complete kinematic decoupling, IEEE T. Automat. Sci. Eng., 9, 538-553, 2012.

Li, Y. M., Wu, Z. G., and Zhao, X. H.: Optimal design and control strategy of a novel 2-DOF micromanipulator, Int. J. Adv. Robot. Syst., 10, 1-13, 2013.

Lin, C. J. and Lin, P. T.: Particle swarm optimization based feedforward controller for a $X Y$ PZT positioning stage, Mechatronics, 22, 614-628, 2012.

Liu, P., Yan, P., Zhang, Z., and Leng, T.: Modeling and control of a novel $X-Y$ parallelpiezoelectric-actuator driven nanopositioner, ISA T., 56, 145-154, 2015.

Mccall, J.: Genetic algorithms for modelling and optimisation, J. Comput. Appl. Math., 184, 205-222, 2005.

Polit, S. and Dong, J.: Development of a high-bandwidth $X Y$ nanopositioning stage for high-rate micro-/nanomanufacturing, IEEE/ASME Transactions on Mechatronics, 16, 724-733, 2011.

Qin, Y., Shirinzadeh, B., Tian, Y., and Zhang, D.: Design issues in a decoupled $X Y$ stage: Static and dynamics modeling, hysteresis compensation, and tracking control, Sensor. Actuat. A-Phys., 194, 95-105, 2013.

Smith, S. T.: Flexures: elements of elastic mechanisms, Gordon and Breach, New York, 2000.

$\mathrm{Su}$, X. P. and Yang, H. S.: Design of compliant microleverage mechanisms, Sensor. Actuat. A-Phys., 87, 146-156, 2001 a.
Su, X. P. and Yang, H. S.: Single-stage microleverage mechanism optimization in a resonant accelerometer, Struct. Multidiscip. O. 21, 246-252, 2001b.

Tang, H. and Li, Y. M.: Design, Analysis, Test of a novel 2-DOF nanopositioning system driven by dual mode, IEEE Transation on Robotics, 29, 650-662, 2013.

Tang, H. and Li, Y. M.: Development and active disturbance rejection control of a compliant micro/nano-positioning piezo-Stage with dua-mode, IEEE T. Ind. Electron., 61, 1475-1492, 2014.

Tang, H. and Li, Y. M.: A new flexure-based $Y \theta$ nanomanipulator with nanometer-scale resolution and millimeter-scale workspace, IEEE/ASME Transation on Mechatronics, 20, 1320-1330, 2015.

Tian, Y., Shirinzadeh, B., and Zhang, D.: A flexure-based mechanism and control methodology for ultra-precision turning operation, Precis. Eng., 33, 160-166, 2009.

Wang, F., Li, J., Liu, S., Zhao, X., Zhang, D., and Tian, Y.: An Improved Adaptive Genetic Algorithm for Image Segmentation and Vision Alignment Used in Microelectronic Bonding, IEEE/ASME Transation on Mechatronics, 19, 916-923, 2014.

Wu, Z. G. and Li, Y. M.: Optimal design and control strategy of a novel 2-DOF micromanipulator, Int. J. Adv. Robotic Syst., 10, 1-13, 2014.

Yong, Y. K., Aphale, S. S., and Moheimani, S. O.: Design, Identification, and Control of a Flexure-Based $X Y$ Stage for Fast Nanoscale Positioning, IEEE Transation on Nanotechnology, 8 , 46-54, 2009.

Yu, J., Xie, Y., Li, Z., and Hao, G.: Design and Experimental Testing of an Improved Large-range Decoupled $X Y$ Compliant Parallel Micromanipulator, J. Mech. Robot., 7, 044503, https://doi.org/10.1115/1.4030467, 2015.

Yu, Y. Q., Du, Z. C., Yang, J. X., and Li, Y.: An Experimental Study on the Dynamics of a 3-RRR Flexible Parallel Robot, IEEE Transation on Robotics, 27, 992-997, 2011. 